SCIENTIFIC REPORT

\title{
Suprachoroidal haemorrhage complicating cataract surgery in the UK: epidemiology, clinical features, management, and outcomes
}

\author{
R Ling, M Cole, C James, S Kamalarajah, B Foot, S Shaw
}

Br J Ophthalmol 2004;88:478-480. doi: 10.1136/bjo.2003.026138

\begin{abstract}
Aims: To study the incidence, management, and outcomes of suprachoroidal haemorrhage (SCH) complicating cataract surgery in the United Kingdom.

Methods: Cases were prospectively collected by active surveillance through the British Ophthalmological Surveillance Unit. Details were obtained using an incident questionnaire with follow up at 6 months.

Results: 118 cases were reported in 1 year. The estimated incidence of $\mathrm{SCH}$ was $0.04 \%$ (95\% confidence interval $0.034 \%$ to $0.050 \%$ ). Cataract extractions were by phacoemulsification in $76.2 \%$, extracapsular cataract extraction (ECCE) in $11.0 \%$, and phacoemulsification conversion in $12.8 \%$. SCH was "limited" (1 to 2 quadrants) in $48.7 \%$, "full blown" ( 3 to 4 quadrants) in $43.1 \%$. SCH in phacoemulsification was more likely to be limited (63.2\%), compared with ECCE (11.1\%) and phacoemulsification conversion (23.1\%) $\left(p<0.001, \chi^{2}\right.$ test). Visual acuity (VA) was better than $6 / 60$ in 57 of $95(60 \%)$ cases after a median follow up interval of 185 days. 33 of 34 cases $(97.1 \%)$ with secondary anterior segment revision had VA better than 6/60. VA was worse than $6 / 60$ in 7 of $8(87.5 \%)$ cases that had intraoperative sclerostomy, and in all $6(100 \%)$ cases that had secondary posterior segment intervention.

Conclusion: $\mathrm{SCH}$ is a rare but serious complication of cataract surgery. Poor prognostic factors included full blown $\mathrm{SCH}, \mathrm{ECCE}$, phacoemulsification conversion, retinal apposition, and retinal detachment.
\end{abstract}

$\mathrm{S}$ uprachoroidal haemorrhage $(\mathrm{SCH})$ is a potentially devastating complication of cataract surgery. Epidemiological data are limited to a few retrospective personal and institutional series, some over 15 years old..$^{1-6}$ The more recent national cataract surgery survey estimated the incidence of $\mathrm{SCH}$ to be $0.1 \%$, although $23 \%$ of cataract operations were performed by the extracapsular technique (ECCE).

The present study was undertaken to provide current data on epidemiology, management, and outcomes of $\mathrm{SCH}$ complicating cataract surgery in a prospective national survey.

\section{MATERIALS AND METHODS}

\section{Case definition}

Suprachoroidal haemorrhage cases were defined as haemorrhage in the suprachoroidal space during cataract surgery, diagnosed by the surgeon. Cases were excluded when cataract extraction was combined with another intraocular procedure.

\section{Bosu reporting system}

New cases were prospectively ascertained through the monthly active surveillance system of the British Ophthalmological Surveillance Unit $(\mathrm{BOSU})^{8}$ from November 2000 to October 2001. All permanently employed ophthalmologists with clinical autonomy in the UK (consultants and associate specialists) received a reporting card at the end of each month containing up to five different conditions. They were asked to indicate any new cases of these conditions (in this case $\mathrm{SCH}$ ), or to confirm that they had no new cases to report. Following positive notification, reporting ophthalmologists were sent a baseline questionnaire requesting medical and ophthalmic history, intraoperative details, and postoperative findings of the $\mathrm{SCH}$ cases. Information on subsequent management and outcome was sought in a follow up questionnaire 6 months later.

\section{Assessment of case ascertainment}

The completeness of case ascertainment among ophthalmologists who contributed cases was assessed through a comparison of BOSU reports with the hospital critical incident databases (which record serious complications such as $\mathrm{SCH}$ ) from ophthalmologists who reported cases during the first 3 months of the study.

\section{RESULTS}

\section{Case notifications}

A total of 176 cases were notified; 162 (92.0\%) questionnaires were returned. There were 118 new cases, 10 duplicates, and 34 incorrect diagnoses.

\section{Incidence of $\mathrm{SCH}$}

Only NHS cases were included for the incidence calculation, 109 of which were reported. During this period, the total number of cataract extractions performed within the NHS, excluding combined procedures, was estimated to be 260000 (to the nearest 1000). ${ }^{9}$ This produces an estimated incidence of $0.04 \%$ (95\% confidence interval $0.034 \%$ to $0.050 \%$ ), or 1 in 2400 (to the nearest 100) cataract extractions.

\section{Case ascertainment}

Although not directly linked to ascertainment rates, the response rate is commonly used to assess the potential for selection bias. ${ }^{10}$ The mean BOSU card return rate during our study period was $72 \%$, with mean response rates ranging from $67 \%$ to $88 \%$ from all UK regions. ${ }^{11}$ This represents a

Abbreviations: BOSU, British Ophthalmological Surveillance Unit; ECCE, extracapsular technique; $\mathrm{SCH}$, suprachoroidal haemorrhage; $\mathrm{VA}$, visual acuity 
period of consistently high card return rate since the establishment of BOSU in 1997.

Fourteen "validation centres" provided a total of 13 cases on their hospital critical incident databases. These centres did not differ significantly in terms of hospital type (district/ teaching) or regional distribution from all UK national. All 13 cases were reported to BOSU. In addition, there were nine BOSU notifications from the validation centres that were not recorded on their critical incident databases.

\section{Patient details}

Of the 118 cases, nine were confirmed as SCH but further information was not made available. Baseline information was therefore available for 109 cases. The mean age was 82 years (range 54-98), with 74\% female. Cataract extractions were under local anaesthesia in $97.2 \%$, and by surgeons of consultant grade in $70.4 \%$. There were $83(76.2 \%)$ phacoemulsification, 12 (11.0\%) ECCE, and 14 (12.8\%) phacoemulsification conversions. Posterior capsule was ruptured in 57 $(52.3 \%)$ cases.

\section{Intraoperative diagnosis and management}

Signs of SCH are summarised in table 1 . There is an increasing tendency for $\mathrm{SCH}$ to manifest as the procedure progressed, reaching a peak during irrigation/aspiration (fig 1). The diagnosis of SCH was suspected intraoperatively and subsequently confirmed in 14 cases.

Prompt wound closure was achieved without any additional manoeuvres (other than using viscoelastic to deepen the anterior chamber) in $63(57.8 \%)$ cases. Additional intraoperative manoeuvres included posterior sclerostomy in eight $(7.3 \%)$, excision of prolapsed iris in $19(17.4 \%)$, and anterior vitrectomy in $35(32.1 \%)$.

\section{Extent of $\mathrm{SCH}$}

Clinical findings on the first postoperative assessment following SCH are detailed in table 1. SCH was "limited" (one to two quadrants) in $53(48.7 \%)$, "full blown" (three to four quadrants) in $47(43.1 \%)$, and obscured by media opacity in nine $(8.2 \%)$ cases. SCH was significantly more likely to be limited during phacoemulsification $(63.2 \%$

Table 1 Signs that led to the intraoperative diagnosis of $\mathrm{SCH}$, and the extent of $\mathrm{SCH}$ on the first postoperative examination

\begin{tabular}{lc} 
& \\
& No of cases (\%) \\
\hline Intraoperative signs $(n=109)$ & \\
Shallowing of anterior chamber & $85(78.0 \%)$ \\
Hardening of eye & $65(59.6 \%)$ \\
Loss of red reflex & $40(36.7 \%)$ \\
Bulging posterior capsule & $43(39.4 \%)$ \\
Extrusion of intraocular contents & \\
Iris & $43(39.4 \%)$ \\
Lens & $1(0.9 \%)$ \\
Vitreous & $7(6.4 \%)$ \\
Retina & $3(2.8 \%)$ \\
Postoperative findings ( $=109)$ & \\
Quadrants of SCH ( $=109)$ & $38(34.9 \%)$ \\
One & $15(13.8 \%)$ \\
Two & $8(7.3 \%)$ \\
Three & $39(35.8 \%)$ \\
Four & $9(8.2 \%)$ \\
Unknown & $22(22.2 \%)$ \\
Central retinal apposition $(n=99)$ & $22(20.4 \%)$ \\
Vitreous incarceration to wound $(n=108)$ & $26(24.5 \%)$ \\
Iris incarceration to wound $(n=106)$ & $3(2.9 \%)$ \\
Retinal incarceration to wound $(n=105)$ & $27(25.2 \%)$ \\
Vitreous haemorrhage $(n=107)$ & $21(19.3 \%)$ \\
Retained lens material $(n=109)$ & \\
\hline & \\
&
\end{tabular}

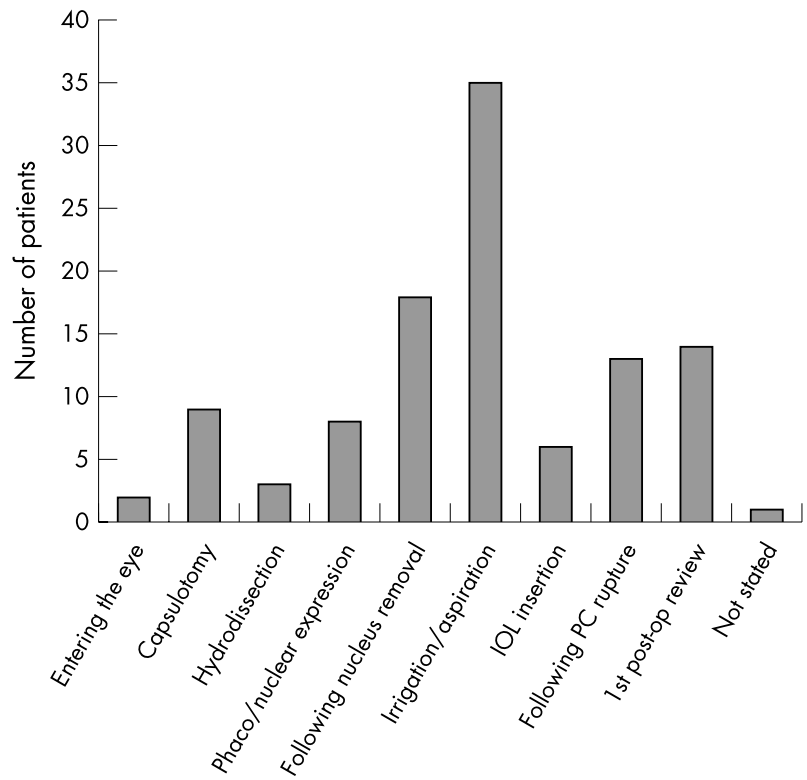

Figure 1 Stages of cataract surgery during which signs of $\mathrm{SCH}$ first became manifest.

limited; $36.8 \%$ full blown) compared with both ECCE ( $11.1 \%$ limited; $88.9 \%$ full blown) and phacoemulsification conversion (23.1\% limited; $76.9 \%$ full blown) $\left(\mathrm{p}<0.001, \chi^{2}\right.$ test).

\section{Postoperative interventions and outcome}

Follow up was available in $100(91.7 \%)$ cases. Excluding five cases with less than 30 days follow up, best corrected visual acuity (VA) was $6 / 12$ or better in $40 \%, 6 / 18$ to $6 / 60$ in $20 \%, 6 /$ 60 or worse in $40 \%$, after a median follow up interval of 185 (55-395) days. In these patients, factors associated with VA $<6 / 60$ include full blown SCH, ECCE, phacoemulsification conversion, retinal apposition, and retinal detachment $(\mathrm{p}<0.001$, Mann-Whitney U test $)$.

There were three main forms of surgical interventions in our series:

- Intraoperative drainage sclerostomy was performed in eight patients. Seven $(87.5 \%)$ had full blown SCH and all had VA less than 6/60 (2 NLP) at the 6 month follow up.

- Secondary anterior segment revisions occurred in 34 patients, all with limited SCH, after an interval of 6 (113) days. Eleven had secondary IOL implantation alone. VA of $6 / 60$ or better at 6 months was achieved in 33 (97.1\%) cases.

- Secondary posterior segment interventions were undertaken in six of 14 cases $(42.9 \%)$ complicated by retinal detachment. All underwent pars plana vitrectomy after an interval of 19 (14-54) days. Retinal reattachment was achieved in four cases. However, all cases with retinal detachment had VA worse than 6/60 at 6 months, regardless of whether they were observed or received secondary surgical management.

\section{DISCUSSION}

This prospective national survey confirms that $\mathrm{SCH}$ is a rare complication of cataract surgery. Surveillance using a single source to identify cases is vulnerable to under ascertainment. ${ }^{8}$ The incidence of $0.04 \%$ should be regarded as a minimum estimate, as is the case with other surveillance studies. ${ }^{12}$ 
However, the consistently high BOSU card return rate and the $92 \%$ reply rate ( 162 of 176 questionnaires returned) both indicate good compliance with the study. More importantly, critical incidence databases from 14 centres indicated that all cases had been reported to BOSU. There are, therefore, good reasons to have confidence in the completeness of reporting.

Our incidence is significantly lower compared with the $0.1 \%$ in the national cataract surgery survey (NCSS). ${ }^{7}$ This is unlikely to be due to under ascertainment for reasons stated above, but represents a change in the actual rate related to changes in surgical technique. The incidence of $0.04 \%$ is closer to previously reported incidence figures in phacoemulsification (Davison ${ }^{4} 0.06 \%$, Eriksson et al $0.03 \%$ ). These authors demonstrated a lower incidence of $\mathrm{SCH}$ in phacoemulsification compared with ECCE. ${ }^{4}$

Most authors consider ocular hypotony to be essential in the development of SCH. ${ }^{13}{ }^{14} \mathrm{SCH}$ occurred most frequently after removal of the nucleus in our study. In phacoemulsification, this necessitates a change of instruments when the relative stability of the closed system is at risk of being temporarily disrupted. In ECCE, nuclear expression causes maximal distortion of the globe, and ocular hypotony is inevitable. Prompt normalisation of intraocular pressure is therefore advisable. In phacoemulsification, this can be achieved by filling the anterior chamber with viscoelastic before starting irrigation/aspiration. In ECCE, we recommend pre-placed sutures in the section to aid reformation of the anterior chamber with viscoelastic after nucleus expression.

Our study confirms previous observations that $\mathrm{SCH}$ tends to be limited in phacoemulsification. ${ }^{6}{ }^{15}$ The self-sealing wound allows rapid closure and provides effective tamponade against the developing $\mathrm{SCH}$, an advantage that is lost in ECCE and conversion. The extent of SCH has significant prognostic implications: cases with full blown $\mathrm{SCH}$ were six times more likely to have final VA of less than 6/60 compared with limited SCH in our series.

Of our cases with intraoperative drainage sclerostomy, $87.5 \%$ had full blown SCH by the next postoperative day and subsequently poor outcome. In a rabbit model of SCH, sclerostomy resulted in marked extension of the $\mathrm{SCH} .{ }^{16}$ Intraoperative sclerostomy cannot therefore be recommended. Immediate closure of the open globe must remain the priority in the intraoperative management of $\mathrm{SCH}$.

The results of secondary anterior segment revision are encouraging. This is largely because they were limited SCH with no significant posterior segment complications. Good results from anterior segment revision have also been reported. ${ }^{217}$

The same cannot be said of secondary vitreoretinal interventions; cases with retinal detachment had uniformly poor outcome whether or not they received secondary posterior segment intervention. Better visual outcome from secondary vitreoretinal intervention has been reported..$^{18}$ However, indications are that retinal detachment complicating SCH in cataract surgery carries a poor prognosis, and that aggressive posterior segment intervention should probably be reserved for "only eye", and a few selected cases with clearly achievable surgical goals.

In conclusion, SCH remains a serious complication of cataract surgery, with potentially devastating consequences to vision. Fortunately the incidence is very low, and a good visual outcome is still possible in limited extent cases that occur during small incision phacoemulsification cataract surgery.

\section{ACKNOWLEDGEMENTS}

This project was made possible by the data contribution of over 100 ophthalmologists throughout the UK, which the authors wish to gratefully acknowledge. In addition, the authors would like to thank the "validation centres" for providing critical incident databases for the external validation of SCH cases.

The authors are very grateful for the support of the British Ophthalmological Surveillance Unit throughout the study. We also wish to thank Mrs Persis Ladwa for her administrative help, Dr Tineke Freni for her constructive advice, and Ellen Farleigh, James Harvey, Hugh James, and Clare Maley for data entry.

This project was supported by the Torbay Medical Research Fund, and the British Council for Prevention of Blindness, for which we are grateful.

\section{Authors' affiliations}

R Ling, M Cole, C James, Department of Ophthalmology, Torbay Hospital, Torquay, UK

S Kamalarajah, Eye and Ear Clinic, Royal Victoria Hospital, Belfast, UK B Foot, The British Ophthalmological Surveillance Unit, The Royal College of Ophthalmologists, UK

S Shaw, Department of Mathematics \& Statistics, University of Plymouth, UK

Correspondence to: MrR Ling, Consultant Ophthalmologist, West of England Eye Unit, Royal Devon \& Exeter Hospital (Wonford), Barrack Road, Exeter EX2 5DW, UK; roland.ling@rdehc-tr.swest.nhs.uk

Accepted for publication 1 September 2003

\section{REFERENCES}

1 Taylor DM. Expulsive hemorrhage. Am J Ophthalmol 1974;78:961-6.

2 Davison JA. Acute intraoperative suprachoroidal hemorrhage in extracapsular cataract surgery. J Cat Refract Surg 1986; 12:606-22

3 Arnold PN. Study of acute intraoperative suprachoroidal hemorrhage. J Cataract Refract Surg 1992;18:489-94.

4 Davison JA. Acute intraoperative suprachoroidal hemorrhage in capsular bag phacoemulsification. J Cataract Refract Surg 1993;19:534-7.

5 Speaker MG, Guerriero PN, Met JA, et al. A case-control study of risk factors for intraoperative suprachoroidal expulsive hemorrhage. Ophthalmology 1991;98:202-10.

6 Eriksson A, Koranyi G, Seregard S, et al. Risk of acute suprachoroidal hemorrhage with phacoemulsification. J Cataract Refract Surg 1998;24:793-800.

7 Desai P, Minassian DC, Reidy A. National cataract surgery survey 1997-8: a report of the results of the clinical outcomes. Br J Ophthalmol 1999;83:1336-1340.

8 Foot B, Stanford M, Rahi J, et al. The British Ophthalmological Surveillance Unit: an evaluation of the first 3 years. Eye 2002;16:1-7.

9 Hospital Episode Statistics (HES), Department of Health (DOH) of England, Wales, Scotland and Northern Ireland, November 2000 to October 2001.

10 Asch DA, Jedrziewski MK, Christakis NA. Response rates to mail surveys published in medical journals. J Clin Epidemiol 1997;50:1129-36.

11 BOSU news. No. 2 Spring, 2002.

12 Kilmartin DJ, Dick AD, Forrester JV. Prospective surveillance of sympathetic ophthalmia in the UK and Republic of Ireland. Br J Ophthalmol 2000;84:259-63.

13 Zauberman H. Expulsive choroidal haemorrhage: an experimental study. $\mathrm{Br} J$ Ophthalmol 1982;66:43-5.

14 Beyer CF, Peyman GA, Hill JM. Expulsive choroidal hemorrhage in rabbits: a histopathologic study. Arch Ophthalmol 1989;107:1648-53.

15 Beatty S, Lotery A, Kent D, et al. Acute intraoperative suprachoroidal haemorrhage in ocular surgery. Eye 1998;12:815-20.

16 Lakhanpal V. Experimental and clinical observations on massive suprachoroidal haemorrhage. Trans Am Ophthalmol Soc 1993;91:545-62.

17 Bryant WR. Secondary intraocular lens implantation in eyes that experienced suprachoroidal hemorrhage during primary cataract surgery. J Cataract Refract Surg 1989;15:629-34.

18 Reynolds MG, Haimovici R, Flynn Jr HW, et al. Suprachoroidal hemorrhage, clinical features and results of secondary surgical management. Ophthalmol 1993;100:460-5. 